\title{
In situ AFM investigation of slow crack propagation mechanisms in a glassy polymer
}

\author{
M. George ${ }^{a}$, Y. Nziakou ${ }^{\mathrm{a}, \mathrm{b}, \mathrm{c}}$, S. Goerke ${ }^{\mathrm{a}}$, A.-C. Genix ${ }^{\mathrm{a}}$, B. Bresson ${ }^{\mathrm{b}, \mathrm{c}}$, S. Roux ${ }^{\mathrm{d}}$, \\ H. Delacroix ${ }^{\mathrm{b}, \mathrm{c}}$, J.-L. Halary ${ }^{\mathrm{b}, \mathrm{c}}$, M. Ciccotti ${ }^{\mathrm{b}, \mathrm{c}}$ \\ ${ }^{a}$ Laboratoire Charles Coulomb, Université Montpellier, CNRS, Montpellier, France \\ ${ }^{b}$ Laboratoire SIMM, CNRS, ESPCI Paris, PSL Research University, Paris, France \\ ${ }^{c}$ Laboratoire SIMM, Université Pierre et Marie Curie, Sorbonne-Universités, Paris, France \\ ${ }^{d}$ Laboratoire LMT, Ecole Normale Supérieure Paris-Saclay, CNRS, Université Paris-Saclay, \\ Cachan, France
}

\begin{abstract}
A novel experimental technique based on in situ AFM monitoring of the mechanisms of damage and the strain fields associated to the slow steady-state propagation of a fracture in glassy polymers is presented. This micron-scale investigation is complemented by optical measurements of the sample deformation up to the millimetric macroscopic scale of the sample in order to assess the proper crack driving conditions. These multi-scale observations provide important insights towards the modeling of the fracture toughness of glassy polymers and its relationship with the macromolecular structure and non-linear rheological properties. This novel technique is first tested on a standard PMMA thermoplastic in order to both evaluate its performance and the richness of this new kind of observations. Although the fracture propagation in PMMA is well known to proceed through crazing in the bulk of the samples, our observations provide a clear description and quantitative evaluation of a change of fracture mechanism towards shear yielding fracture accompanied by local necking close to the free surface of the sample, which can be explained by the local change of stress triaxiality. Moreover, this primary surface necking mechanism is shown to be accompanied by a network of secondary grooves that can be related to surface crazes propagating towards the interior of the sample. This overall scenario is validated by post-mortem fractographic investigations by scanning electron microscopy.
\end{abstract}

Email addresses: matthieu.george@umontpellier.fr (M. George), matteo.ciccotti@espci.fr (M. Ciccotti) 
Keywords: Glassy polymers, Atomic force Microscopy, Fracture, Micromechanims

\section{Introduction}

Glassy polymers are widespread in technical and structural applications since the 30's due to their excellent mechanical properties, combined with low mass density and ease of implementation. Although their elastic moduli and yield strength are lower than other structural materials such as glasses or metals, glassy polymers possess a relatively high fracture toughness. This results from a combination of plastic deformation and the occurrence of several localization mechanisms such as crazing and shear banding (Donald and Kramer, 1982). Extensive studies have been devoted to the modeling of the relation between the mechanical properties and the material composition and microstructure. Their main aim was combining contributions from both macroscopic constitutive laws, mesoscale damage mechanisms and molecular issues (Williams, 1984; Brown, 1991; Réthoré and Estevez, 2013; Halary et al., 2011).

Although glassy polymers (i.e. with a glass transition temperature well above ambient temperature) can belong to different families with different macromolecular structures such as thermoplastics, thermosets and semicrystalline polymers, their macroscopic mechanical properties are qualitatively quite similar up to yield condition. These materials have a typical elastic modulus of $E \approx 1 \mathrm{GPa}$, a yield strength of about $\sigma_{y} \approx 100 \mathrm{MPa}$, and a fracture toughness of about $K_{c} \approx 1$ $\mathrm{MPa} \sqrt{\mathrm{m}}$. The size of the inelastic process zone at the crack tip is thus of the order of magnitude of the Dugdale length:

$$
\ell_{D}=\chi \frac{K_{c}^{2}}{\sigma_{y}^{2}} \approx 15 \div 40 \mu \mathrm{m}
$$

where $\chi$ ranges between $1 / 2 \pi \simeq 0.16$ and $\pi / 8 \simeq 0.39$ depending on the choice of a circular (McClintock and Irwin, 1964) or elongated (Dugdale, 1960) process zone.

It is thus possible to use the tools of Linear Elastic Fracture Mechanics (LEFM) on typical laboratory fracture specimens of centimeter size. Although the materials of this class share similar properties on scales larger than $\ell_{D}$, the nature of the inelastic processes occurring in the process zone can be very different depending on the nature and structure of the macromolecular network, which results in a very different large strain behaviour. The kinetics of crack propagation can also 


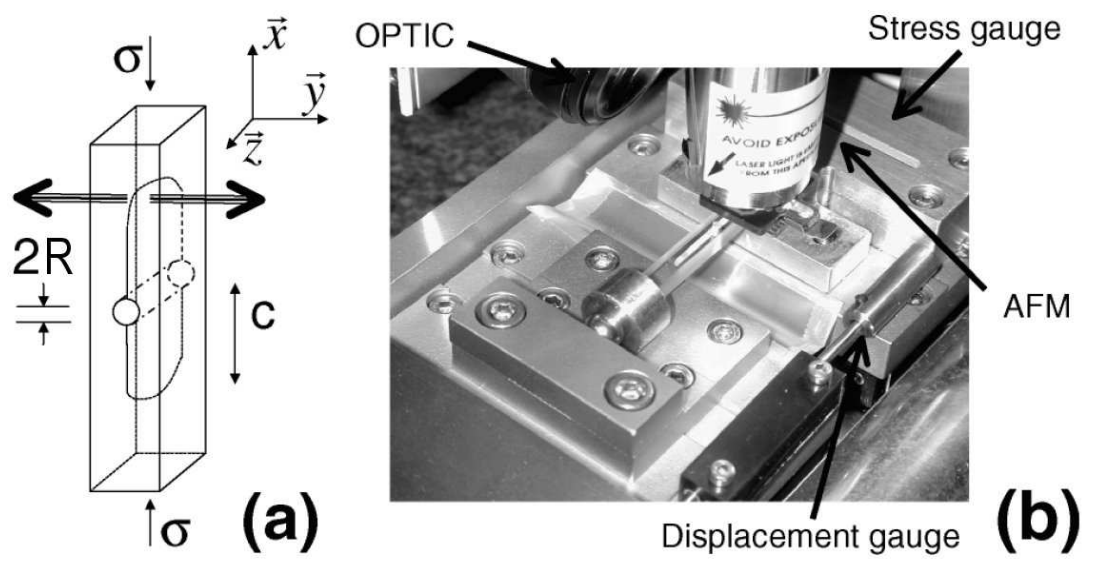

Figure 1: Experimental setup: (a) Sketch of the DCDC geometry; (b) picture of the experiment, including the DCDC sample on a loading stage under the AFM probe.

be very variable, changing from very stable steady-state crack propagation over a wide range of velocities in thermoplastics to jerky stick-slip fracture propagation in thermosets. Understanding and engineering the toughness of these polymers relies on the ability of describing and modeling the bulk deformation and energy dissipation mechanisms at the process zone scale, which is micrometric in size. This makes Atomic Force Microscopy (AFM) in situ investigations an ideal tool.

An adequate description of the deformation mechanisms requires the observation of a well conditioned steady-state propagating fracture. This allows the energy flow to be coherently defined from the macroscopic scale of the loading stage down to the ultimate molecular failure scale. This novel technique exploits the combination of an AFM (Bruker ICON, Santa Barbara, USA) with a very stable and compact in situ loading configuration (Fig. 1) based on the Double Cleavage Drilled Compression (DCDC) sample (Janssen 1974) and a very stiff and compact loading stage (Deben Microtest, Woolpit, UK). The macroscopic nature of the sample and its excellent stability allow obtaining a well defined mode I loading and a very straight crack path. This allows for a very stable steady-state fracture propagation in materials where the fracture toughness $K_{c}$ can be expressed as an increasing function of the crack propagation velocity $v$, such as thermoplastic glassy polymers. The two main constraints arising in this local probe investigation are (1) that crack propagation is limited to slow velocities (between $\mathrm{pm} / \mathrm{s}$ and $\mathrm{nm} / \mathrm{s}$ ) and (2) that observations are limited to the free surface of the sample. This technique was initially developed and extensively studied on more brittle materi- 
als such as oxide glasses (Janssen, 1974; He et al., 1995; Pallares et al., 2009). It has already provided enlightening information on the nanoscale mechanisms of stress-corrosion during sub-critical crack propagation (Ciccotti, 2009; Pallares et al., 2015), including the relevance of crack tip plasticity (Han et al., 2010), stress induced ion exchange processes (Célarié et al., 2007) and capillary condensation in the crack tip cavity (Grimaldi et al, 2008; Pallares et al., 2011). An extension of this technique is presented here for glassy polymers, where the use of DCDC samples has been until recently hampered due to the difficulty of preventing buckling and plastic yielding of the sample, which is loaded in compression (Plaisted et al., 2006).

The present study is focused on the study of PMMA since its mechanical and fracture properties have been widely characterized. Slow crack propagation in PMMA at ambient conditions has long been known to be dominated by crazing on a micrometric region close to the crack tip. This consists of nanometric fibrils being nucleated and drawn across the crack lips and providing extensive energy dissipation and crack propagation resistance (Doll, 1983; Kramer, 1983). However, while the crazing mechanism is well established in the bulk of the sample, i.e. in the center of the crack front, where the plane-strain condition induces a high degree of stress triaxiality ${ }^{1}$, its occurrence at the free surface of the sample is questionable due to the local reduction of the hydrostatic stress (Argon and Hannoosh, 1977; Kramer, 1983; Si et al., 2005). Our in situ AFM observations at the free surface of the sample show evidence of a transition in the fracture mechanism towards shear yielding in a surface layer of about $10 \mu \mathrm{m}$ thickness. While the large 4 $\mathrm{mm}$ thickness of the sample allows the experimental investigation of this interesting change of mechanism in a well conditioned mechanical loading, this boundary effect can become very important for understanding fracture propagation in thin samples of few tens of $\mu \mathrm{m}$ thickness.

The manuscript is organized as follows. Section 2 reports the developments of the DCDC technique required to obtain a well conditioned steady-state crack propagation in glassy polymers. Section 3 reports the optical measurements required to characterize both the crack propagation kinetics and the strategy adopted to estimate the stress intensity factor for partially yielding samples. Section 4 reports the in-situ AFM investigation of the strain fields in a $40 \mu \mathrm{m}$ wide region progressively crossed by the process zone. The observed main necking region

\footnotetext{
${ }^{1}$ Stress triaxiality is defined as $\psi=\sigma_{m} / \sigma_{d}$, where $\sigma_{m}$ is the hydrostatic stress and $\sigma_{d}$ is the equivalent Von Mises stress, which represents the shear stress (Lemaitre and Chaboche, 1990).
} 
and secondary grooves are discussed and interpreted in terms of past knowledge on the physics and mechanics of inelastic processes occurring in the process zone for PMMA. Section 5 provides an overview of the potential of this novel investigation technique on a thermoset polymer. Section 6 drives the conclusions concerning both the novel experimental technique and the interesting observations on the surface features of fracture propagation in PMMA.

\section{Development of the DCDC technique for glassy polymers}

\subsection{Classic fracture mechanics of the DCDC sample for brittle samples}

The DCDC sample was initially developed by Janssen (1974) to study slow fracture in silicate glasses, which possess very high values for both Young modulus $E \approx 70 \mathrm{GPa}$ and yield strength $\sigma_{y} \approx 10 \mathrm{GPa}$. The sample (Fig. 1a) consists of a prism of dimensions $2 L \times 2 w \times 2 t$ (corresponding to $x, y, z$ directions) with a cylindrical cross hole of radius $R$ drilled through the specimen thickness $2 t$. The sample is loaded with a compressive force $F$, and thus a compressive stress $\sigma=F / 4 w t$ applied to the two opposite faces. This induces a tensile stress at the two poles of the central hole aligned with the sample loading direction. During the test in stiff and hard materials like glass, two symmetric cracks of length $c$ are spontaneously nucleated at the crown of the central hole. The two cracks then propagate in opposite directions along the midplane of the sample (direction $x$ in the $x-z$ plane), driven by the mode I opening induced at the crack tip.

In these very brittle materials the sample deformation is essentially linear elastic. The stress intensity factor has been derived by several authors via finite element simulations (Janssen, 1974; Michalske et al., 1993; He et al., 1995; Pallares et al., 2009). When the crack tip is at a sufficient distance ( $>w)$ from the central hole and the sample ends, the mode I stress intensity factor can be expressed in the general form:

$$
K_{I}=\sigma \sqrt{R} f\left(\frac{c}{R}, \frac{w}{R}\right)
$$

where $f$ is a decreasing function of the crack length $c$ and the sample width $w$. Several different analytical approximations have been proposed for the function $f$ by the authors cited above. Crack propagation is thus very stable at both imposed load and imposed displacement $\left(d K_{I} / d c<0\right)$. Moreover, the DCDC configuration presents an excellent directional stability of the straight crack propagation. This is due to the strongly negative values the $T$ stress induced at the crack tip by the applied compression (Fett et al., 2005). 
The toughness of materials presenting sub-critical crack propagation is characterized by a $K_{I c}(v)$ with positive slope as a function of the velocity $v$ up to a critical velocity $v_{c}$ where crack propagation undergoes an instability towards dynamic propagation. When using the DCDC sample to study sub-critical propagation with a constant applied displacement, the crack progressively slows down as a consequence of the increase of the crack length. This allows to progressively reach very slow velocities without any activation of the loading stage. At the very low crack propagation velocities required by AFM observations (below 10 $\mathrm{nm} / \mathrm{s}=36 \mu \mathrm{m} / \mathrm{h}$ ), the variations of the crack length $c$, and consequently of $K_{I}$, are very weak. A steady-state propagation in very pure asymptotic mode I loading $\left(K_{I I} / K_{I} \leq 5 \%\right)$ can thus be observed over conveniently long in situ investigations : a few days or weeks depending on the crack propagation velocity.

\subsection{Crack initiation for glassy polymers and geometrical optimization}

The application of the DCDC configuration to glassy polymers implies several issues since these materials are less stiff $(E \approx 1 \mathrm{GPa})$ and less hard $\left(\sigma_{y} \approx 100\right.$ $\mathrm{MPa}$ ) than silicate glasses. Moreover they present a slow viscoelastic relaxation at macroscopic scale. These materials are not brittle enough to provide a spontaneous crack initiation from the central hole. Even when the two cracks are well established, their steady-state propagation should be attained while limiting as much as possible the buckling and yielding of the sample at macroscopic scale. Last, but not least, the estimation of the stress intensity factor acting at the crack tip is made considerably more difficult by the occurrence of stress relaxation, larger deformations and plastic yield due to the shear stress concentration in the region of the central hole.

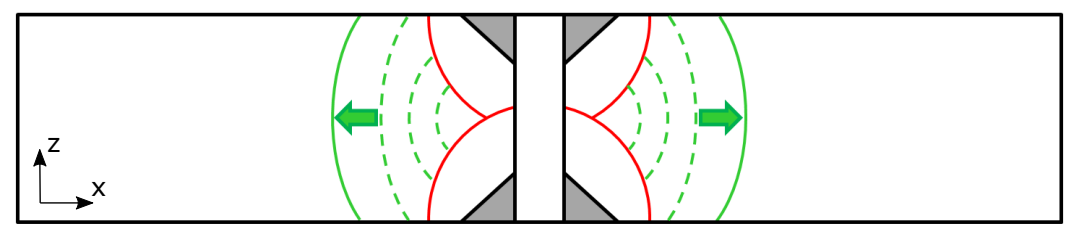

Figure 2: Two step initiation of the DCDC cracks in glassy polymers. The indented triangular blades (grey) induce the pop-in of the two penny shape cracks (red). The loading of the DCDC sample induces the development of the two parabolic propagating cracks (green).

The crack initiation was implemented with the help of an instrumented razor blade (Nziakou, 2015) by developing the techniques proposed by Idonije et al. (1993) and Plaisted et al. (2006). In a first step, two sharp triangular blades were 
indented at each side of the central hole up to obtaining pop-in of two penny shape cracks that merge in the middle of the sample (in red in Fig. 2). In a second step the DCDC sample is preloaded in its standard configuration in order to obtain the full coalescence of the penny cracks. This leads to the development of the two typical DCDC cracks with parabolic crack front (in green in Fig. 2).

In order to minimize buckling and yielding during crack propagation while preserving the small sample dimension required by our in situ AFM investigation, the scaling of Eq. (1) allows to optimize the sample dimensions. PMMA has a similar toughness $K_{I c}$ to glass, but lower values for the stiffness and yield strength. Eq. (1) suggests that the $K_{I} / \sigma$ ratio can be increased by increasing the radius $R$ of the central hole. To preserve the scaling of eq. (1), the sample width $2 w$ and the crack length $c$ should also be increased in a proportional way. The sample thickness $2 t$ is not implied in eq. (1), so we used the maximum value allowed by our loading stage. The sample dimensions selected for the present investigation are $2 L=45 \mathrm{~mm}, 2 w=7.2 \mathrm{~mm}, 2 t=4.95 \mathrm{~mm}$ and $R=1.2 \mathrm{~mm}$. The stable propagation of very straight cracks with slow velocities in the desired range for AFM investigation was achieved, presenting steady-state conditions over several days.

\subsection{Strategies of evaluation of $K_{I}$ for glassy polymers}

The rest of this section is dedicated to the discussion of the evaluation of $K_{I}$ on DCDC samples made of glassy polymers by treating in an independent way the occurrence at the macroscopic scale of stress relaxation, finite strain and plastic yield of the sample.

\subsubsection{Macroscopic stress relaxation}

In the case where macroscopic stress relaxation is the only issue, the relaxation following the initial loading of the sample up to a fixed displacement can be treated by considering an elastic modulus $E(t)$ that weakly diminishes with time. In this case, equation (1) can be used for the calculation of $K_{I}$ from a direct measurement of the applied force F (Marshall et al., 1974). From a practical point of view, for very slow propagating cracks, this can lead to a progressive reduction of $K_{I}$ (and thus of the crack velocity) even when the variation of the crack length is not appreciable on a macroscopic scale.

\subsubsection{Large strain in $D C D C$ samples}

Since the values of $K_{I}$ for crack propagation in silicate and polymer glasses are quite similar, while the elastic modulus of glassy polymers is about 100 times 
smaller, the strain field and the associated displacements are 100 times larger. In particular, the compressive displacement applied to the sample is about $1 \mathrm{~mm}$ and the crack opening at the intersection with the central hole can reach $1 \mathrm{~mm}$, which leads to an appreciable deformation of our samples (cf. Fig. 3), and makes the use of the linear equation (1) questionable. An alternative modeling has been proposed by Plaisted et al. (2006) based on the elastic buckling of the two beams surrounding the full crack length (outward buckling in direction $y$ in Fig. 3). This model results in $K_{I}$ values that are proportional on the applied stress $\sigma$, but independent of the crack length $c$. While the predicted values of $K_{I}$ are comparable to those given by eq. (1) for short cracks, they are larger for longer cracks. This model was tested by Plaisted et al. (2006) on crack arrest tests on PMMA, but it does not provide consistent values in our steady-state tests, and remains questionable since both relaxation and yielding are neglected.

\subsubsection{Local strategy to handle plastic yielding in DCDC samples}

If the plastic yielding was limited to the the process zone of micrometric size close to the crack tip, small scale yielding conditions would allow the use of LEFM and eq. (1) for the calculation of $K_{I}$ on our centimeter sized DCDC samples. However, in the case of a plastic yield on a more macroscopic region the nonlinear structural response of the DCDC sample should be evaluated by a different elasto-plastic modeling. Due to the stress concentration induced by the presence of the central hole, both the plastic yield and large strains remain confined in the two ligaments around the central hole, as confirmed by empirical considerations on our DCDC samples (cf. Fig. 3). The effect of a plastic yielding of the ligaments around the central hole would lead to almost constant bending moments applied to each of the elastic dual cantilever systems, and thus to an equation for $K_{I}$ that is once again almost independent on the crack length (Hutchinson and Suo, 1992). Based on these considerations, we derive here a technique for the estimation of $K_{I}$ based on a local measurement of the crack opening profile $u_{y}(X)$ over an intermediate scale range where the material response is neither affected by crack tip inelastic processes nor by the plastic yield around the hole. LEFM solutions are thus applicable to evaluate the value of $K_{I}$ applied to the process zone from these intermediates scale measurements.

In a previous work (Pallares et al., 2009) it was shown by finite element simulation that for an elastic DCDC sample, the crack opening profile $u_{y}(X)$ over a distance from the crack tip less than the specimen half-width $(X<0.85 w)$ can be written in terms of a Williams expansion with invariant coefficients for all the 


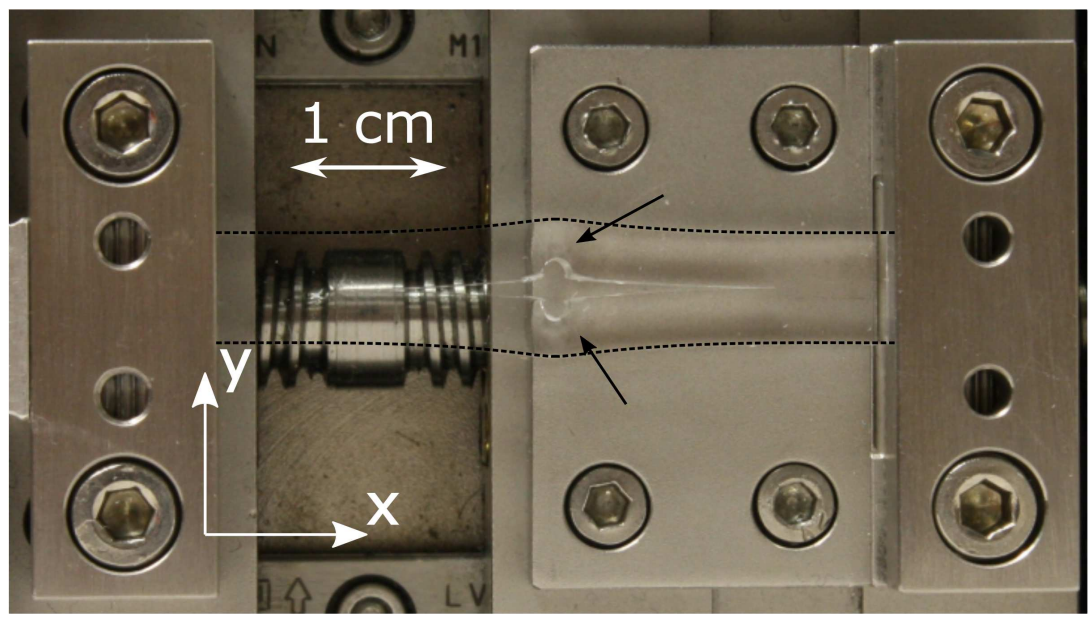

Figure 3: PMMA DCDC sample under load. The black arrows indicate local large strain regions close to the central hole.

211

specimen geometries and crack lengths analyzed:

$$
u_{y}(X)=p \sqrt{X}\left(1+1.319\left(\frac{X}{w}\right)+0.515\left(\frac{X}{w}\right)^{2}\right)
$$

$$
p=\frac{K_{I}}{E^{\prime}} \sqrt{\frac{8}{\pi}}
$$

where $E^{\prime}=E /\left(1-v^{2}\right)$ because plane-strain conditions are dominant in our specimen. Since the region of validity of this expression ( $2 \mathrm{~mm}$ here) remains elastic in our PMMA specimens, we can extend its application to our experiments for evaluating the stress intensity factor from the crack opening displacement measured in this intermediate region. In the presence of a significant stress relaxation, the evaluation of $K_{I}$ from the crack opening profile would require a suited evaluation of the relaxation modulus $E(t)$.

Since the relative importance of stress relaxation, finite strain and plastic yield can change between different glassy polymers, the choice of the appropriate method for the estimation of $K_{I}$ should be evaluated and discussed in each case. In the present paper the application to PMMA will be discussed in detail based on our experimental results and on the comparison with values reported in the literature. 


\section{Crack propagation results of PMMA samples}

This section will be devoted to the application of the techniques developed to obtain a controlled crack propagation in DCDC samples made of PMMA, and to perform an appropriate evaluation of the corresponding values of $K_{I}$. The measured $K_{I c}(v)$ curve is then validated against measurements available in the literature for similar grades of PMMA.

\subsection{Material properties and crack propagation kinetics}

The material used is a commercial grade of PMMA with very low additive content. The glass transition temperature is $T_{g}=122^{\circ} \mathrm{C}$ as measured by Differential Scanning Calorimetry (DSC), the average molecular weight is $M_{w}=1200$ $\mathrm{kg} / \mathrm{mol}$ with polydispersity 1.7 as measured by Gel Permeation Chromatography (GPC), the density is $\rho=1180 \mathrm{~kg} / \mathrm{m}^{3}$ after Nziakou (2015).

During our experimental campaign we could obtain a successful crack initiation and well conditioned steady-state crack propagation over four different samples of PMMA. The quality of the crack propagation was assessed by evaluating the degree of symmetry of the crack along each of the three symmetry axes of the DCDC sample: weak deviation $(y<100 \mu \mathrm{m})$ of the crack plane from the mid plane $(x z)$, small difference in length between the two propagating cracks $(\Delta c<100 \mu \mathrm{m})$, good centering and weak tilt of the parabolic crack fronts (the position where the green fronts in Fig. 2 intersect the two external surfaces should not differ more than $100 \mu \mathrm{m}$ ). This latest point is a very sensitive test against the occurrence of buckling in a direction $y$ perpendicular to the loading direction $x$. Despite the optimization of the sample dimensions, a close inspection of our loaded DCDC samples reveals the presence of macroscopic bulk plastic yielding in the region of the central hole, with an extension limited to the millimetric size of the hole (cf. Fig. 3). Crack propagation was performed at ambient conditions $\left(T=23 \pm 2^{\circ} C, R H=35 \pm 10 \%\right)$. By applying forces ranging between 900 and $1200 \mathrm{~N}( \pm 4 \mathrm{~N})$ crack propagation velocities were spanned over a large range $\left(10^{-10} \mathrm{~m} / \mathrm{s}\right.$ to $\left.10^{-5} \mathrm{~m} / \mathrm{s}\right)$ measured by combining in situ AFM and optical microscopy observations (cf. Fig. 4). Each sample provided a very large amount of measurements over the available range of crack length $c$ between 4 and $12 \mathrm{~mm}$.

\subsection{Evaluation of $K_{I}$ for PMMA samples}

In light of the macroscopic yield region observed in the neighborhood of the central hole (cf. Fig. 3), $K_{I}$ was estimated using the local strategy presented in section 2.3.3, based on the measurement of the crack opening profiles. Since the 


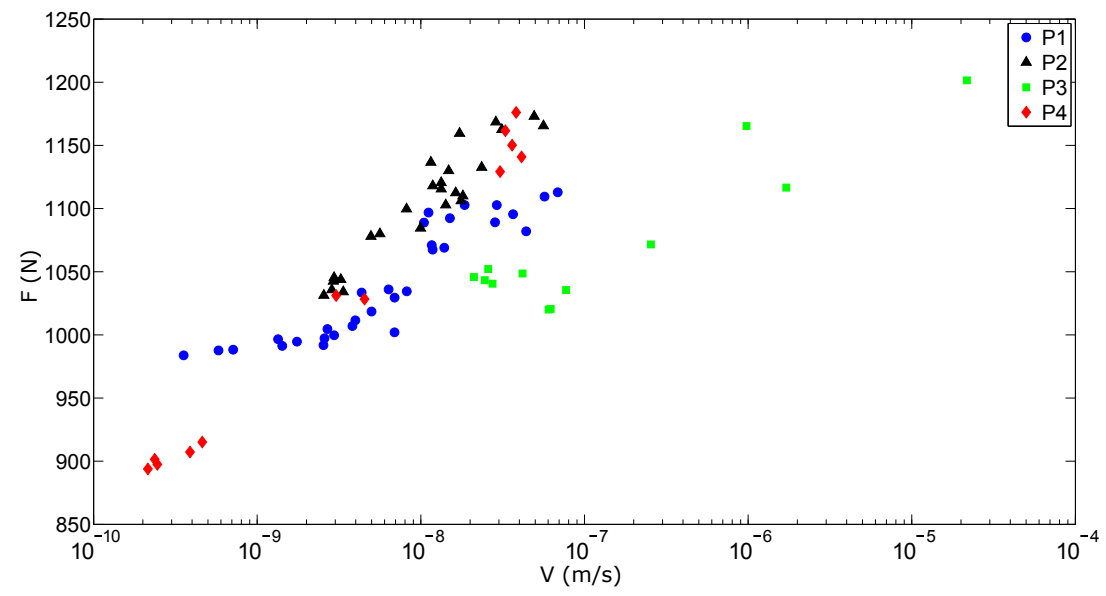

Figure 4: Measurements of the crack velocities and applied forces for the four different samples (named in the legend). Most measurements of crack velocities are performed by optical microscopy on series of $140 \mu \mathrm{m}$ sized images. The velocities below $5.10^{-9} \mathrm{~m} / \mathrm{s}$ are measured by AFM on series of 40 or $10 \mu \mathrm{m}$ wide images.

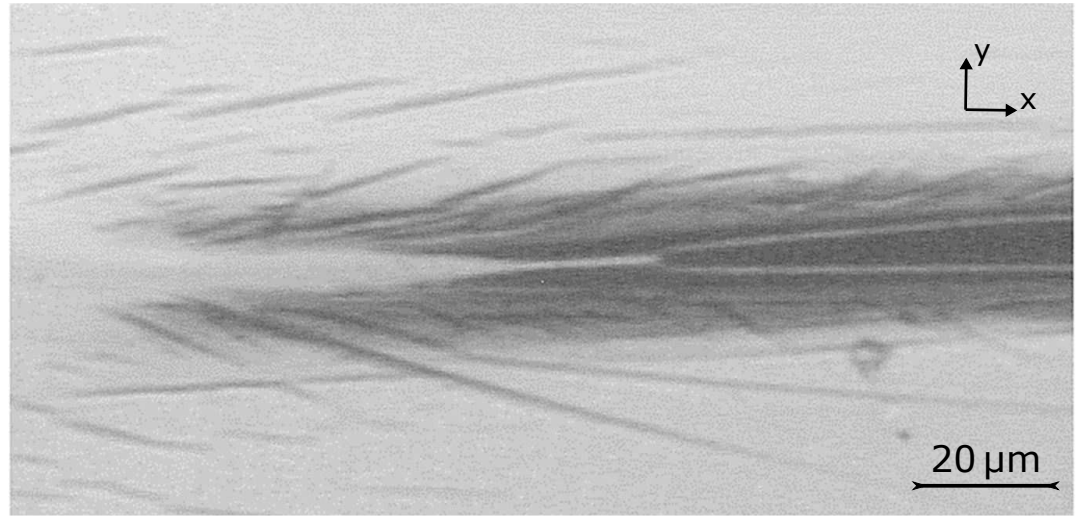

Figure 5: Neighborhood of the crack tip imaged by light reflection. The crack propagates towards the left of the image ( $x$ direction). The crack opening profile in the right of the picture is identified by the bright line surrounding the black crack cavity. The elongated dark and bright regions surrounding and extending the crack cavity represent en extended necking region as confirmed by AFM measurements in section 4.2. The dark lateral portions represent strongly tilted side walls where the incident light is reflected out of the camera objective, while the bright regions represent flat zones that cause direct light reflection into the camera objective. The gray tilted lines represent secondary surface grooves as confirmed by AFM measurements in section 4.3. A full movie corresponding to several hours of steady state propagation can be found in the supplementary information (SI). 


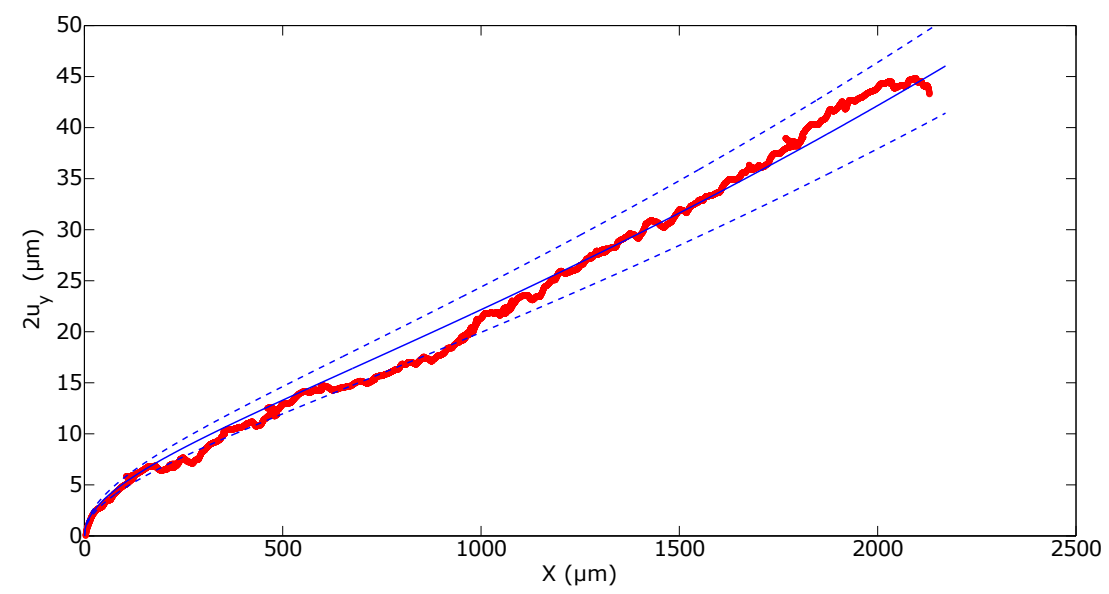

Figure 6: Crack opening profile measured by optical microscopy (red line), calculated as the vertical distance $2 u_{y}$ between the two bright lines surrounding the black crack cavity in Fig. 5 as a function of the distance $X$ from the crack tip. The blue line represents the least square linear fit through eq. (2) and the blue dashed lines represent $\mathrm{a} \pm 10 \%$ variation on the fitted parameter $p$.

sample width is $2 w=(7.2 \pm 0.1) \mathrm{mm}$, the crack opening profiles $u_{y}(X)$ were measured over the region of validity $X<2 \mathrm{~mm}$ of the Williams expansion (Eq. 2) starting at the crack tip. Because of the very small crack opening $(<100 \mu \mathrm{m})$ the measurement was performed by combining several overlapped optical microscope images of $140 \mu \mathrm{m}$ width of the free surface of the sample, such as the one in Fig. 5. The crack opening profile was measured by detecting the position of the bright line surrounding the open crack. A calibration of this observable against AFM measurements over the accessible region of $300 \mu \mathrm{m}$ from the crack tip (cf. Nziakou, 2015) allowed to obtain accurate crack opening profiles such as the one plotted in Fig. 6. The obtained profiles are well described by the fit through the Williams expansion of Eq. (2) (blue line in Fig. 6), which has a single adjustable parameter $p$ related to its opening. The precision on the measurement of $p$ can be estimated to be $\pm 10 \%$. This is due to the limited resolution of the optical images, which are perturbed by the presence of the extended damaged zone at the surface of the DCDC sample. Since the expected variations of $K_{I c}$ for PMMA over the measured slow velocity range is very weak (cf. data from Doll, 1983, reported in Fig. 7), the precision of the local crack opening technique reveals to be insufficient for an accurate measurement of the dependence of $K_{I c}$ on $v$.

On the other hand, the resolution of the force measurements is clearly higher $( \pm 4 \mathrm{~N})$ and their correlation with the velocity measurements is clearly apparent, as observed in Fig. 4. The choice was made to use a hybrid approach to take 
the best party of all available measurements: (1) using the measured crack opening profiles to set the average value of $K_{I}$ during all our experiments on the four PMMA samples reported in Fig. 7 and (2) using the force measurements in order to estimate the slope of the $K_{I c}(v)$ curves reported in Fig. 7. The assumption of the proportionality between $K_{I}$ and $F$ is consistent with both the arguments of Plaisted et al. (2006) and Hutchinson and Suo (1992) that support a weak or vanishing dependence of $K_{I}$ over the crack length $c$ due to large strain and yield near the central hole of the sample.

In order to relate the average measured opening $\langle p\rangle=(4.8 \pm 0.5) \cdot 10^{-4} \mathrm{~m}^{1 / 2}$ to the average stress intensity factor $\left\langle K_{I}\right\rangle$ through Eq. (3), an appropriate value of the elastic modulus has to be estimated. Since our crack propagation tests are very slow and cover several days, it is appropriate to chose the relaxed modulus of PMMA at ambient temperature. This can be estimated by the Dynamic Mechanical Analysis (DMA) measurement at $1 \mathrm{~Hz}(E=2.4 \pm 0.2 \mathrm{GPa}, v=0.35 \pm 0.03$, according to Nziakou, 2015) since most of the $\beta$ relaxation of PMMA occurs at higher frequencies in the $100 \mathrm{~Hz}$ range and further long term relaxation can be estimated to less than $10 \%$ (Halary, 2011). The average stress intensity factor obtained through eq. (3) is $\left\langle K_{I c}\right\rangle=(0.8 \pm 0.1) \mathrm{MPa} \mathrm{m}^{1 / 2}$. The $K_{I c}(v)$ curve plotted in Fig. 7 is thus obtained by renormalizing the force data in Fig. 4 by the relation $K_{I c}=F\left(<K_{I c}>/<F>\right)$, where $\langle F>=(1000 \pm 100) \mathrm{N}$ is the average value of the measured force over the same set of data. The $K_{I c}(v)$ data from the literature are also plotted to show the good consistency within the expected $10 \%$ accuracy.

The precision of the technique should be improved in the future for providing more accurate measurements of the $K_{I c}(v)$ curves of glassy polymers, which only present weak velocity dependence at ambient temperature. However, the main purpose of this section is to prove that we can robustly obtain a well conditioned steady-state slow crack propagation regime and to control the crack propagation over several orders of magnitude of velocity. This insures the good conditions for the in situ AFM investigations presented in the next section.

\section{AFM investigation}

\subsection{Evaluation of the strain fields in the process zone}

\subsubsection{Qualitative observation of the AFM data}

Optical microscopy (cf. Fig. 5 and movie in SI) revealed that steady-state crack propagation is accompanied by a whole range of localized damage processes at the free surface of the sample, extending over a large neighborhood of the crack tip. To get a more accurate observation of the process zone and of this secondary 


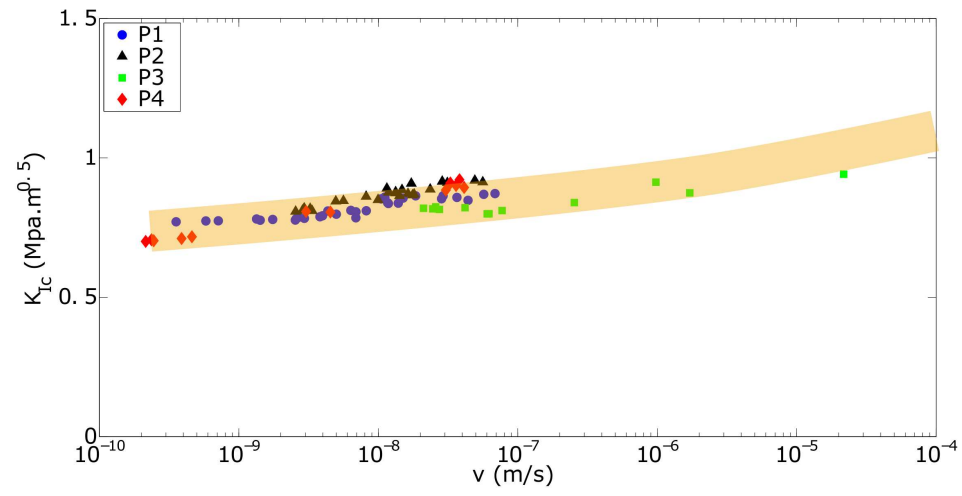

Figure 7: $K_{I c}(v)$ data for PMMA obtained by the present technique compared with data from Doll (1983), within the estimated 10\% accuracy (orange band).

damage zone occurring at the free surface of the DCDC sample (cf. Fig. 1), AFM images of $40 \times 40 \mu \mathrm{m}^{2}$ size were acquired in contact mode ${ }^{2}$ in the crack tip region providing topographic images such as shown in Fig. 8. The height resolution of $0.2 \mathrm{~nm}$ is very small in front of the height variations in the crack tip region, which approach the maximum height range of $7 \mu \mathrm{m}$ achievable on a single AFM image. The lateral resolution is generally provided by the size of AFM tip, which is nominally of $10 \mathrm{~nm}$, but it can quickly be worn to a few tens of $\mathrm{nm}$ during imaging. However in our $40 \mu \mathrm{m}$ wide images $\left(512 \times 512\right.$ pixels $\left.^{2}\right)$, it is limited by the $78 \mathrm{~nm}$ size of the pixels.

On the one hand, the black region surrounding the crack tip in the optical

\footnotetext{
${ }^{2}$ An AFM image is obtained by scanning a cantilever provided with a very sharp tip over a series of parallel lines that progressively form an image. When imaging in contact mode the AFM is in molecular repulsive contact with the sample surface and the deflection of the cantilever is measured to estimate the applied force. During the scan of each line, a feedback loop acts on the vertical position of the AFM head in order to keep the deflection of the cantilever constant to a given set point (Binnig and Quate, 1986). The resulting vertical position of the head during the scan is used to form a height image, which is essentially a topographic image, provided the sample is stiff enough like PMMA. A second kind of image is also provided by recording the error signal of the feedback loop, i.e. the difference between the measured deflection signal and its setpoint value. In case of perfect imaging this error image should be flat and close to zero. On a practical point of view, this image contains both the instrumental noise $(0.2 \mathrm{~nm})$ and a signal which is closely related to the derivative of the height image along the scanning direction. These images are particularly useful to highlight fine surface imperfections while getting rid of smooth height variations.
} 
images (cf. Fig. 5) can be seen as resulting from an extended "necking" of the free surface of the sample (a few micrometer deep), inside which a sharp crack tip is propagating. On the other hand, the network of secondary dark lines that were apparent on the optical images can be clearly interpreted as a series of minor surface grooves of very shallow depth and small width superimposed to the main necking region. Note that the very fine lines that can be distinguished on the AFM images are surface scratches due to polishing of the sample surface prior to the mechanical test.

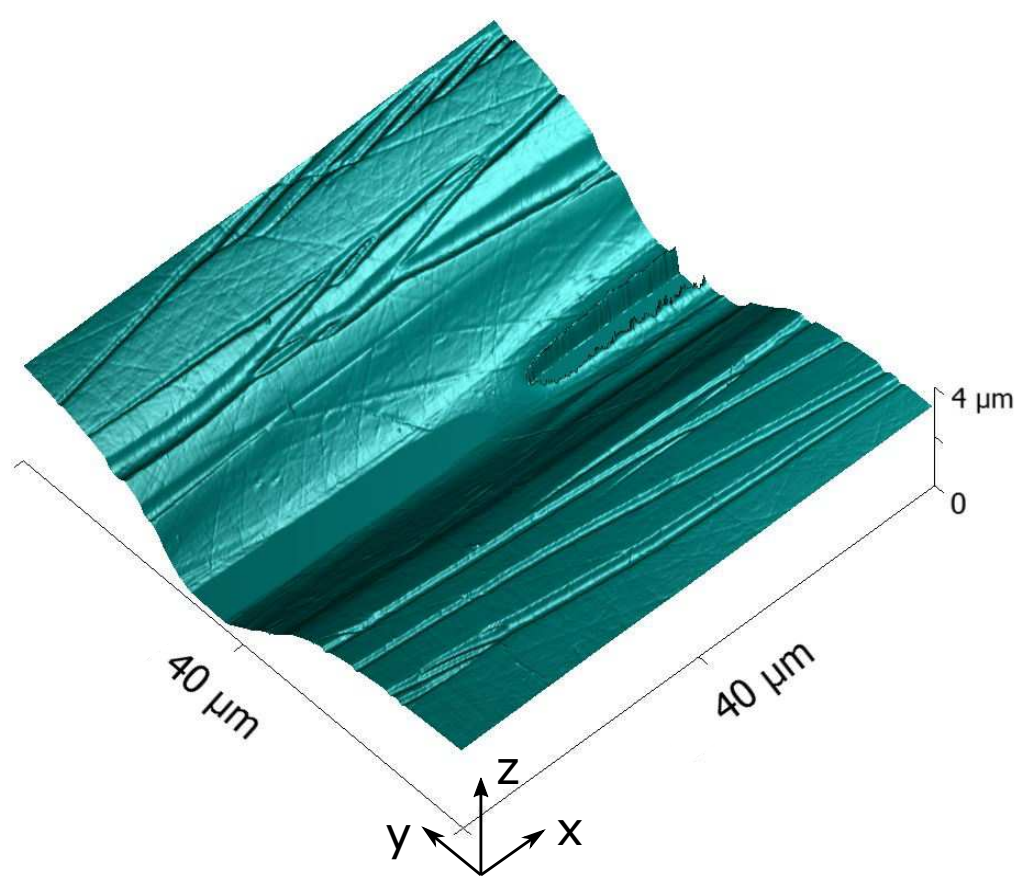

Figure 8: 3D representation of the topography of the crack tip region $\left(40 \mathrm{x} 40 \mu \mathrm{m}^{2}\right)$ measured by AFM at the free surface of the sample. Note that the aspect ratio of the vertical axis is exaggerated by a factor 2 for better appreciating the topography of the necking region.

The optical measurements reveal that the surface necking region at the free surface of the sample extends about $300 \mu \mathrm{m}$ ahead of the observed crack tip, which is significantly larger than the typical $30-40 \mu \mathrm{m}$ size generally observed for the craze region in the middle of the sample (Doll, 1983). However, an enhancement and potential modification of the mechanisms of crack plasticity at the free surface should be expected (Kramer, 1983). In order to proceed to a more systematic characterization of this extended process zone region over its whole size, we po- 
sitioned the AFM scanning head in an initially unyielded region $300 \mu \mathrm{m}$ ahead of a crack tip propagating at a few nm/s. A series of 150 AFM images of $40 \times 40$ $\mu \mathrm{m}^{2}$ size (the largest scan size providing sufficient spatial resolution) was then acquired every 15 minutes over a period of about 36 hours without moving the scanning head position. The imaging ended after the crack tip had passed through the imaing region and traveled $300 \mu \mathrm{m}$ ahead of it. Fig. 9 presents six of these images taken at different times during the propagation. The full movie can be found in the supplementary information (SI). As detailed in footnote 2, the AFM height images allow appreciating the real shape of the imaged surface, while the error images provide flattened images that highlight fine surface features. During the crack propagation, the main central necking gradually develops as a large valley in front of the crack tip, about $20 \mu \mathrm{m}$ wide and $4 \mu \mathrm{m}$ deep. The secondary grooves develop symmetrically on the two sides of the main necking and propagate forward along with the region of first formation of the central necking (about 270 $\mu \mathrm{m}$ ahead of the crack tip). They thus appear to accommodate the surface stretch that results along the banks of the main necking as its bed sinks down, as it will be discussed in more details in section 4.3. It is worth noting that when exploring the close neighborhood of the crack tip with small size high resolution AFM images there is no clear evidence of fibrillar structures associated to crazing or to other types of damage. However, this is not a strong proof because it is difficult to ensure that the AFM tip doesn't wear to a radius comparable to the interfibril spacing of about $30 \mathrm{~nm}$.

Both the optical and AFM observations of the process zone region have been repeated on the four PMMA samples presented in Fig. 7 and provided the same kind of features. In the next two subsections we present a detailed analysis on the sample P4 in order to extract more quantitative information on the strain fields.

\subsubsection{Characterization of the steady-state out-of-plane displacement field}

A progressive characterization of the plastic deformation field at different scales is now discussed. The extreme stability of the steady-state propagation of the crack in our experimental set up allowed to maintain a well conditioned crack propagation over the 36 hours of measurements $(\langle V\rangle=5 \pm 3 \mathrm{~nm} / \mathrm{s})$.

A great care was taken in reducing AFM drifts (less than $10 \mathrm{pm} / \mathrm{s}$, corresponding to $2 \mu \mathrm{m}$ drift over the three day measurements). All the images could thus be recombined together to obtain a complete and accurate map of the out-of-plane displacement field (direction $z$ ) of the free surface (Fig. 10) over a $640 \mu \mathrm{m}$ region centered at the crack tip. This region contains both the full necking region in front 

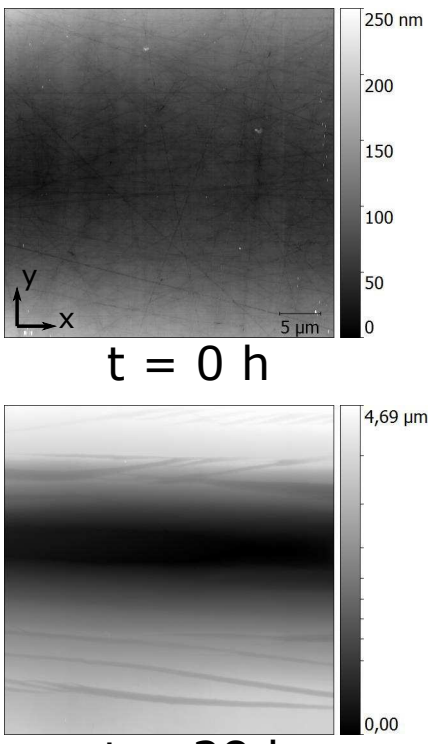

$\mathrm{t}=28 \mathrm{~h}$

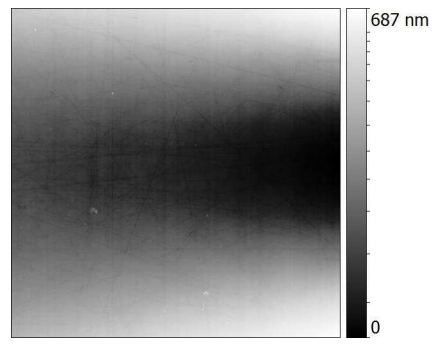

$\mathrm{t}=9 \mathrm{~h}$

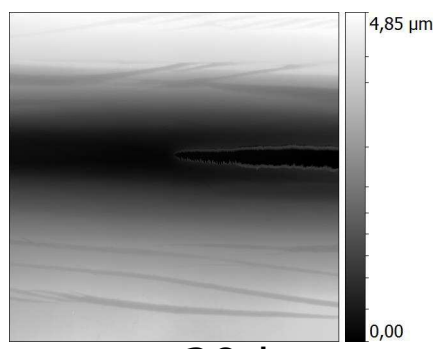

$\mathrm{t}=29 \mathrm{~h}$

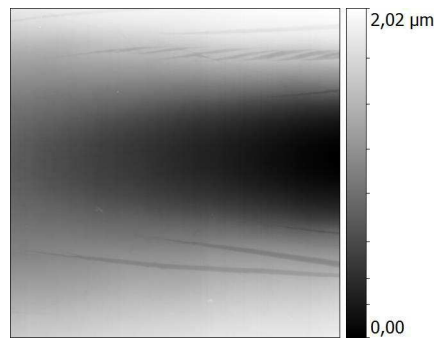

$\mathrm{t}=16 \mathrm{~h}$

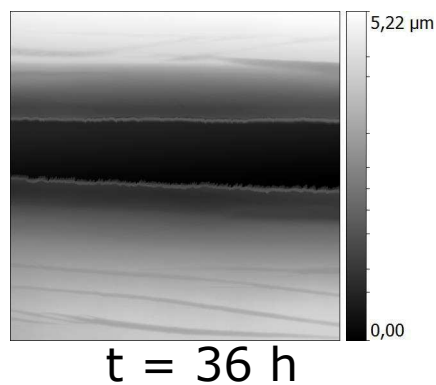

Figure 9: AFM topographical (height) images acquired at 6 different times $t$ during the crack propagation. The full 150 frames movie is available in the SI.

of the crack tip and a portion of the crack opening profile. ${ }^{3}$ It is worth mentioning that because the initial surface was flat, this displacement field is also the mean topography in the reference frame of the crack tip. Since the large displacement field associated to the central necking is propagating along with the crack, the lateral grooves, which are stuck in the material reference frame after their formation, are averaged out by the reconstruction procedure.

The first important remark is that the depression of the free surface caused by the crack tip stresses has a very elongated shape, extended over $300 \mu \mathrm{m}$ ahead of the crack tip and with a lateral span of only $20 \mu \mathrm{m}$. This is very different

\footnotetext{
${ }^{3}$ The acquisition time of each AFM scan line (perpendicular to the crack axis) is combined with the measurement of the crack propagation velocity in order to determine precisely the distance $X$ of the given line from the crack tip position. The full field reconstruction in Fig. 10 results from the juxtaposition of all the measured profiles. Each portion of the steady-state displacement field is thus measured several times on different images. More precisely, since the crack tip field moves in average of $4.5 \mu \mathrm{m}$ in the $x$ direction between two images, there is a $89 \%$ overlap between consecutive images. Each region of the steady-state displacement field is thus measured in average 8 to 9 different times, except near the boundaries of the image series, i.e. in the more distant regions from the crack tip.
} 


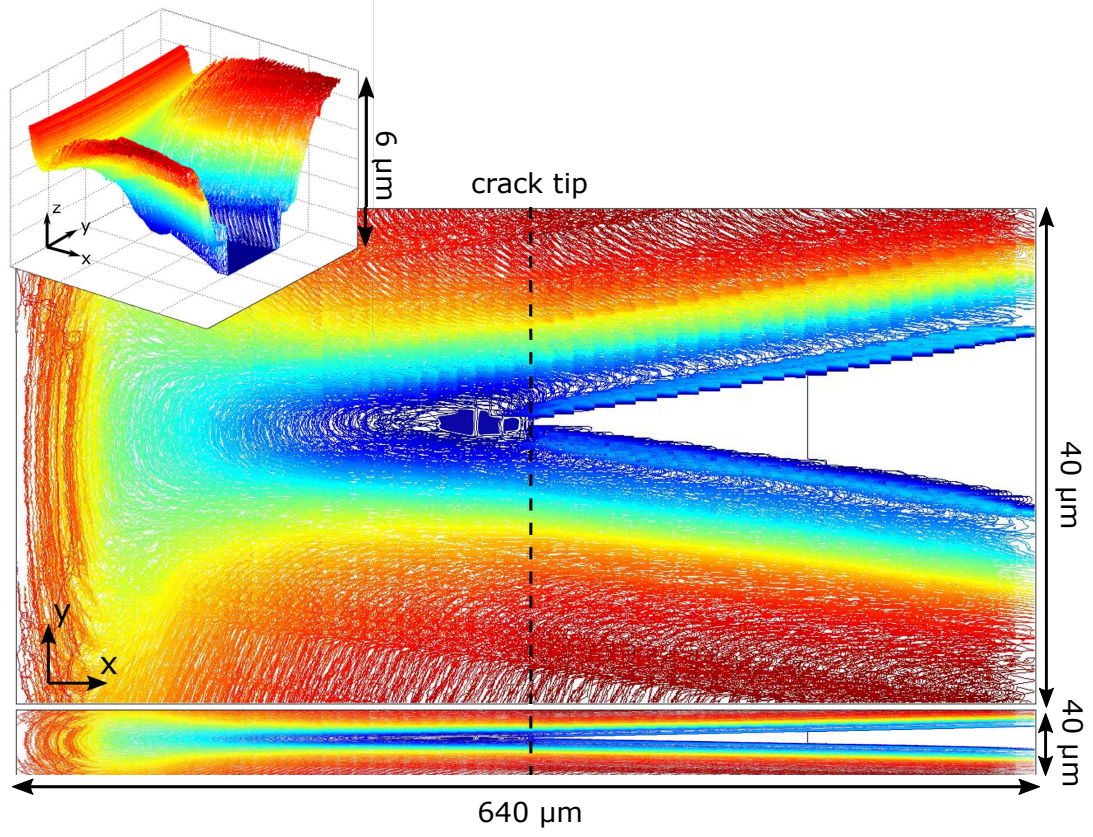

Figure 10: AFM reconstruction of the out-of-plane displacement field of the free surface around the crack tip, top view (the color bar spans $6 \mu \mathrm{m}$ ). The left insert represents a 3D view to appreciate the vertical scaling. Bottom insert represents the top view with the real aspect ratio. It should be noticed that the blue saturated region ahead of the crack tip in the central figure corresponds to the maximum measurable depth allowed by the vertical scan size of the AFM during a uniform image series.

from what was previously observed on DCDC samples made of silicate glasses (Pallares et al., 2015), where the surface depression region has a more rounded shape centered at the crack tip on a millimetric scale. In the case of silicate glasses, comparison with numerical simulations and AFM measurements showed that the surface depression can be fully described by linear elastic crack tip fields down to $10 \mathrm{~nm}$ from the crack tip (Han et al., 2010). The change of shape as revealed by the out-of-plane surface displacement field observed in PMMA involves large strains at a much larger scale $(\sim 100 \mu \mathrm{m})$. The "permanence" of this surface shape change at the crack lips can be seen through the fact that the height depression remains constant after the crack tip has passed through and the associated stresses are released along the crack lips. In other words, the topography on the right of the crack tip in Fig. 10 is a single function of the distance to the crack lips (invariance along the direction that corresponds to the crack opening). This demonstrates clearly the dominantly plastic nature of this necking region in PMMA. Although 
the length of this necking region is much larger than the $30-40 \mu \mathrm{m}$ extension of the crazing region reported in the literature, the surface depression presents a maximum depth at a comparable distance from the crack tip, which frequently induces a local saturation of the AFM topographic signal (cf. blue saturated region in Fig. 10). Moreover, the surface depression presents a slight broadening in the terminal part of the main necking region, located between 200 and $300 \mu \mathrm{m}$ ahead of the crack tip, more akin to the expected behavior from linear elasticity.

\subsection{3. $2 D$ analysis of the in-plane displacement field}

Since the free surface of the sample is initially flat, the in situ topographic AFM images (the traditional height images) directly provide with the vertical component $u_{z}$ of the crack tip displacement field. In order to obtain the in-plane components $\left(u_{x}, u_{y}\right)$ of the crack tip displacement field it is convenient to get rid of the large out-of-plane dynamics $u_{z}$ of the images. This can be done by using the deflection error signal of the feedback loop that is used for AFM imaging in contact mode (cf. the footnote 2 and Binnig and Quate, 1986). The obtained images are much flattened and allow for a good visualization of the surface defects (cf. Fig. 11 and the full movie in the SI), which mainly consist of a dense array of crossed polishing lines. These lines are initially straight (Fig. 11 left), and they constitute a very convenient surface marking to track the in-plane surface deformations along the whole series of AFM images (cf. Fig. 11 right).
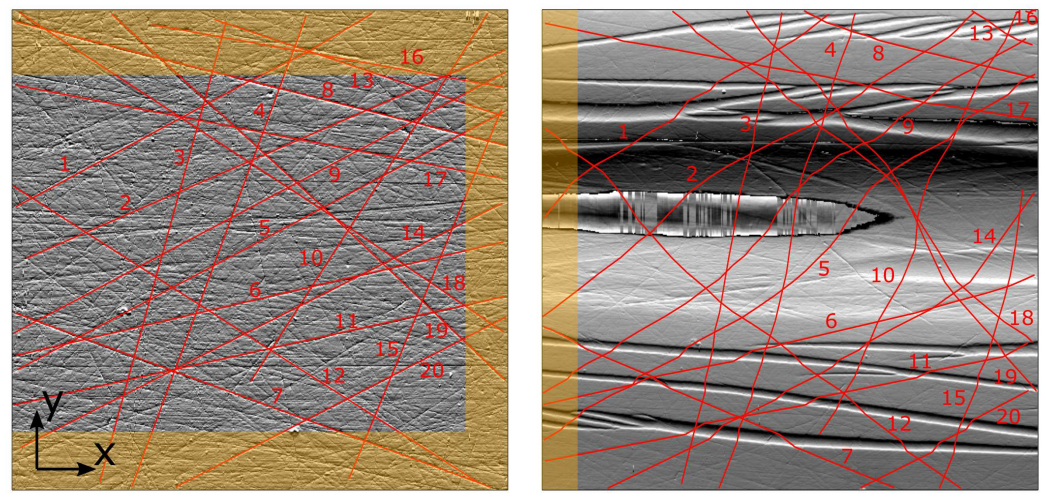

Figure 11: Measurement of the strain field by tracking the shape of the polishing lines network. A limited series of remarkable lines has been highlighted in red and numbered in order to allow for an easier interpretation of the complex deformation field. Note that, once again, the AFM saturation region is visible in the middle of the right image, but this does not limit the tracking of individual lines, which vehiculates the kinematic information. 
In order to provide a straightforward measurement of the $2 \mathrm{D}$ projected strain tensor averaged over a gage length of about $2 \mu \mathrm{m}$, it is convenient to select a series of triplets of remarkable points, each one constituting an initially orthogonal basis in the undeformed reference frame. In practice, we check all intersections between polishing lines in the first (undeformed) image of the series until we identify some suitable triplets that correspond to a couple of segments aligned on the $x$ and $y$ directions and with a length close to the chosen gage length, as represented in black and red in the inset of Fig. 12. The change of length and orientation of the base vectors of a triplet are then monitored through the whole image series as a function of the distance $X$ of the center of the gage region from the crack tip and of the distance $Y_{0}$ of the center of the gage region from the symmetry axis of the crack. When the gage region is not intersected by the secondary grooves, these data can be expressed in terms of the in-plane $(x-y)$ nominal strain field (averaged over the micrometric gage length of the triplet). Fig. 12 presents the evolution of the normal strain component $\varepsilon_{y y}(X)$ (extension in the direction normal to the crack axis) for two such triplets centered at different distances from the crack axis $\left(Y_{0}=0.2\right.$ and $\left.2 \mu \mathrm{m}\right)$. At large distances from the crack tip $(X<-150 \mu \mathrm{m})$ the strain is well below the detection limit (about 5\%). For both curves, the nominal strain presents a significant rise starting at about the same distance of $150 \mu \mathrm{m}$ and then follows an approximately linear trend with distance. For the triplet that is the closest to the crack axis $(0.2 \mu \mathrm{m}$ offset was chosen in order for the whole triplet to belong to the same side of the crack opening) the nominal strain keeps raising linearly up to the crack tip. At this position a large extension of about $\varepsilon_{y y}=70 \%$ (corresponding to a Hencky logarithmic strain $\left.\varepsilon_{H}=\log \left(1+\varepsilon_{y y}\right)=0.5\right)$ is reached, which remains frozen in the region behind the crack tip. Since the scatter in the data corresponds to $5 \%$ strain, which is comparable to the elastic strain limit of PMMA (Monnerie et al., 2005), the measured strain field can be safely attributed to irreversible plastic deformations arising in a process zone region extending 150 $\mu \mathrm{m}$ ahead of the crack tip. The longitudinal strain component $\varepsilon_{x x}$ (extension along the direction of the crack axis) did not present any significant trend out of the 5\% scatter that constitutes the detection limit. It was thus not plotted in the figure for better clarity (data can be found in Nziakou, 2015). The second triplet centered at a larger $2 \mu \mathrm{m}$ offset from the crack axis - displays a similar trend for the nominal strain $\varepsilon_{y y}(X)$. But the saturation takes place at a larger distance from the crack tip $(\sim 80 \mu \mathrm{m})$ and thus for a lower nominal strain value $(\sim 20 \%$, $\left.\varepsilon_{H}=0.18\right)$. This manual image correlation technique is thus very efficient for identifying the plastic process zone and to evaluate the plastic strain field inside it. It should be emphasized that the $150 \mu \mathrm{m}$ length of the plastic process zone is 
only half of the wide $300 \mu \mathrm{m}$ valley seen in the topography surrounding the crack tip (Fig. 10). In the remaining part of the necking region, the material strain is very small and elastic, except in the thin secondary grooves that will be described further down, in section 4.3.

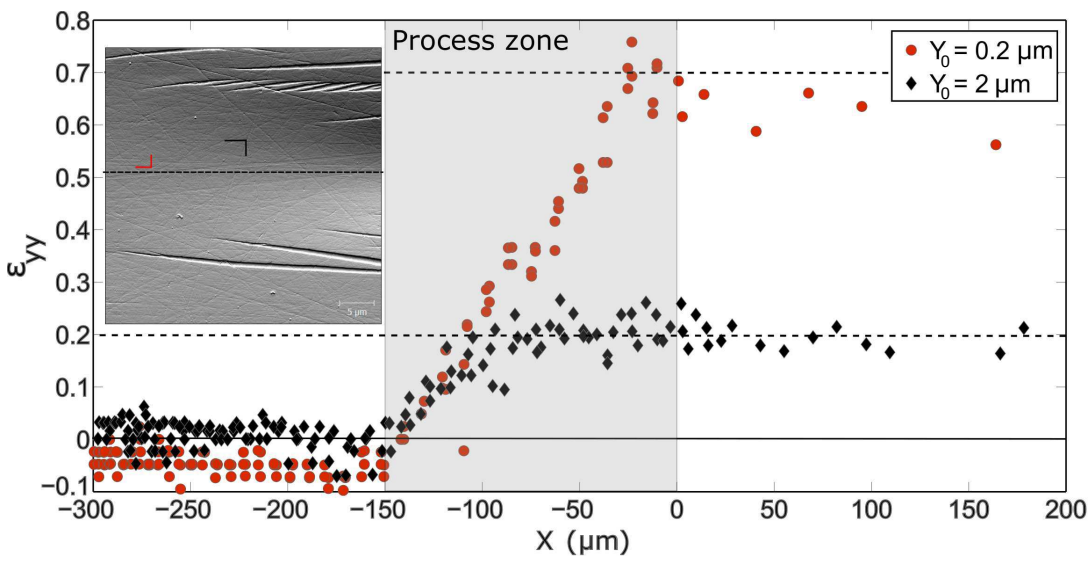

Figure 12: In plane nominal strain field in the direction normal to the crack as a function of the distance $X$ from the crack tip and for two values of the initial offset $Y_{0}$ from the central axis of the main necking region.

\subsection{Interpretation of the main necking region}

The main necking region that has been observed by both optical microscopy and AFM to extend over $300 \mu \mathrm{m}$ length and $20 \mu \mathrm{m}$ width is now discussed. We combine here the information on the surface strain field obtained by the tracking of the polishing lines (Fig. 11) and the surface plastic strain profiles in the process zone ahead of the crack tip (Fig. 12). The main necking region presents essentially along its surface a uniaxial extension field normal to the crack axis with a slow and smooth evolution from the small strain elastic values $(<5 \%)$ out of the process zone size of $150 \mu \mathrm{m}$ length up to the maximum measured values of $70 \%\left(\varepsilon_{H}=0.5\right)$ in the close neighborhood of the crack tip. The curved nature of the strained polishing lines (cf. Fig. 11) shows that the strain field in the main necking region is also smooth in the direction $y$ transverse to the crack propagation direction.

Not only this plastic region is 5 times larger than what expected for a craze propagating in PMMA, but the features of the strain field itself are essentially different from the typical craze field. The strain field in a craze should abruptly change when crossing the craze boundaries from a weak elastic value to a large strain of $100-400 \%\left(\varepsilon_{H}=0.7-1.6\right)$ with a quite uniform distribution inside 
the process zone (Doll, 1983; Kramer and Berger, 1990). If this information is combined with the extended surface depression observed in Fig. 10, the nature of this plastic region at the free surface of the sample should rather be attributed to a bulk shear yielding of the surface region.

The transition in plastic deformation mechanisms from crazing in the inner parts of the sample to shear yielding in the free surface layer can be explained as an effect of the progressive change in triaxiality of the stress field. The inner part of the sample is governed by plane-strain conditions, where the crack front region presents a very high hydrostatic traction. This favors the nucleation of nanometric cavities, which are the precursor for the formation of the fibrillar regions of crazes. The normal stress component $z$ vanishes when approaching the external surface of the sample. The hydrostatic part of the stress field and thus the stress triaxiality are significantly reduced, so that shear yielding at constant volume is favored with respect to cavitation and crazing (Kramer, 1983; Si et al., 2005). The plane-strain extension $\varepsilon_{y y}$ of the craze region in the bulk of the sample is accompanied by a local volume expansion of the material in the same proportion as the nominal strain (factor 2 to 5 after Doll, 1983 and Kramer, 1983). On the other hand, the occurrence of constant volume shear yielding in the surface region implies that the extension $\varepsilon_{y y}=70 \%\left(\varepsilon_{H}=0.5\right)$ in the normal direction to the crack axis, without any measurable $\varepsilon_{x x}$ deformation, is compensated by an equivalent contraction $\varepsilon_{z z}$ in the out-of-plane direction normal to the free surface. This is at the origin of the observed large surface necking region.

A first rough estimation of the depth of the surface layer affected by shear yielding can be obtained by imposing the conservation of volume. In the crack tip region the main necking has a width of about 20 microns and a depth of 4 microns. Since the measured stretches in the $x-y$ plane are $\lambda_{y}=1+\varepsilon_{y y}=1.7$ and $\lambda_{x}=1$, the stretch in the vertical direction should be $\lambda_{z}=1 / \lambda_{y} \simeq 0.6$. The depth of the region affected by the shear yielding can thus be estimated to about $b=10 \mu \mathrm{m}$, as sketched in the left part of Fig. $13 .{ }^{4}$ We remark that the for a $4 \mathrm{~mm}$ thick sample, the inner part of the sample affected by classic crazing mechanism (denoted as $c$ in the figure) constitutes the large majority of the thickness.

In order to test this scenario, a post-mortem investigation on the edge regions of the crack surfaces was performed by Scanning Electron Microscopy (SEM) (right part of Fig. 13). For the four investigated PMMA samples, this systemati-

\footnotetext{
${ }^{4}$ In this rough estimation we neglected the effect of elastic deformation. In light of the large plastic stretch, this can induce a bias of about $10 \%$, i.e. $1 \mu \mathrm{m}$.
} 
cally reveals a marked change in the apparence of the fracture surfaces at a depth of $9 \mu \mathrm{m}$, that confirms a change in the fracture mechanism. A sound interpretation of the lighter hue of the surface layer is the increased roughness of the fracture surfaces produced by tearing under shear yielding. This can induce a stronger electric charge concentration on the asperities, which results in a stronger SEM signal.

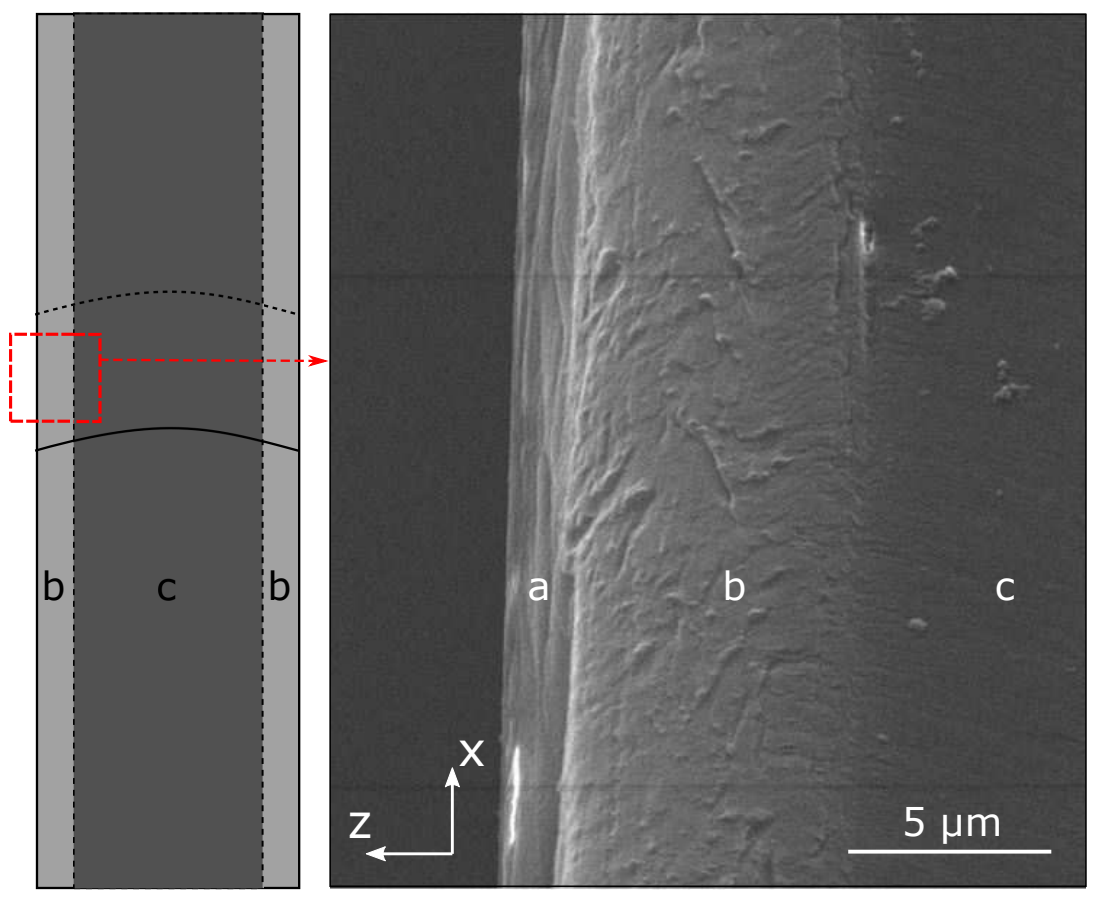

Figure 13: The sketch on the left represents the change of fracture mechanism at a depth $b$ on the fracture surface ( $x-z$ plane, the parabolic crack front propagated in the upward direction). The SEM image on the right corresponds to the region highlighted in red, close to the external sample surface. In the first $9 \mu \mathrm{m}$ region (denoted as $b$ ) the fracture surface properties are remarkably different from the rest of the fracture surface (denoted as $c$ ) indicating a change in the fracture process. The small region $a$ on the extreme edge of the sample represents a side vision of the surface necking region observed by AFM in Fig. 8.

¿From the point of view of mechanics the characteristic $10 \mu \mathrm{m}$ depth where the change of mechanism is observed can be interpreted by the following arguments. It is well known that in materials dominated by shear yielding all over the crack front, like polycarbonate, the progressive loss of triaxiality when approaching the free surfaces has the effect of modifying the shape and size of the shear yielding process zone over a depth equivalent to the size of the process zone itself (as mea- 
sured in the inner plane-strain region, cf. Williams (1984), sec. 5.3) ${ }^{5}$. For those materials, the effect of the loss of stress triaxiality at the surface is simply that of increasing the extension of the process zone and enhancing the local material toughness towards the higher value that is measured on thin samples, where the plane-stress condition is dominant. The effective toughness of a thick sample can thus be estimated with a good approximation by a weighted average between the plane-strain and plane-stress values of the toughness. The weight factor is given by the ratio between the thickness of the altered surface layer (comparable to the Dugdale length) and the total thickness of the sample (Williams, 1984).

In the case of PMMA, the reduction of stress triaxiality involves both a change of mechanism from crazing to shear yielding and a change of the process zone size. The shear necking region presents an approximately five fold increase of the crack opening displacement $h_{C O D}$ and a five fold reduction of the maximum extension $\varepsilon_{\max }$. Since for both mechanisms the fracture energy is approximately given by the plastic work $\Gamma \sim h_{C O D} \sigma_{y} \varepsilon_{\max }$, and the shear yielding stress is quite close to the crazing stress (Halary, 2011), the resulting estimation for the fracture energy does not change significantly. This is also supported by the substantial continuity of the shape of the crack front (measured by characteristic crack arrest markings on the fracture surfaces) when crossing the transition region between the two fracture mechanisms. According to the arguments given above for the transition in the shear yielding process zone size, the depth of the transition from crazing to plastic yield in PMMA should be driven by the $30 \mu \mathrm{m}$ extension of the crazing region in the bulk of the sample. This is quite close to the $10 \mu \mathrm{m}$ order of magnitude observed here, and constitutes a sound mechanical argument for a change of mechanism at a similar scale. To go beyond this rough predictions would require a full 3D finite element modeling of the elasto-plastic deformation of the DCDC sample including large yielding strains and crazing, which is out the scope of the present work.

It should be noted that for our millimeter thick samples the presence of these micrometric surface layers does not affect significantly the effective toughness. On the other hand, a stronger perturbation should be expected when the thickness of the sample is reduced close to the transition range.

\footnotetext{
${ }^{5}$ This can be explained by the fact that the depth of transition of triaxiality is governed by the lateral scales where the stress field is changing, so that for a crack tip singular field the transition depth is progressively shifted towards the free surface when considering smaller and smaller lateral regions close to the crack tip, down to the size of the process zone where the yield onsets.
} 


\subsection{Interpretation of the secondary grooves}

Once the nature of the main necking region has been clearly identified, the network of secondary grooves can be more easily interpreted. They develop symmetrically on both sides of the main necking region and their propagating tips are $270 \mu \mathrm{m}$ ahead of the crack tip, i.e. in the region where the main necking starts to develop. They thus appear to be a secondary surface crazing phenomenon driven by the local stretching normal to the propagation axis induced by the elastic necking at the surface of the sample. This hypothesis is also supported by a finer description of their associated plastic strain field.

Considering the snapshots of AFM error signal images presented in Fig. 14 for two different times of the secondary grooves formation (separated by 3 hours), the initially straight polishing lines are abruptly tilted when they enter the secondary grooves and recover the same direction on the opposite side. This feature reveals the homogeneity of the strain field inside these grooves and the change in angle, which remains constant once the groove is formed, can be used -knowing that the longitudinal displacement in $\mathrm{x}$ direction is null ${ }^{6}$ - to estimate the strain level to very large values between 400 and $500 \%\left(\varepsilon_{H}=1.6-1.8\right)$, while the surrounding regions remains essentially unstrained. When accounting for all the features of our images, the lateral resolution for the angle estimation can be estimated to 5 pixels, i.e. $400 \mathrm{~nm}$.

Thanks to this information on the homogeneity of the strain field inside the secondary grooves, we can extract some important complementary information from the manual digital image correlation applied to a triplet that is progressively crossed by one of these grooves that propagates parallel to the direction $x$ of the main crack axis, as shown in Fig. 15.

In order to obtain a better estimate for the average nominal strain $\varepsilon_{y y}$ inside these localization bands, the measured displacement difference $\Delta u_{y}$ of the two ends of the vertical segment crossing a secondary groove was divided by an estimation $h_{S G}$ of the unstrained width of the secondary groove, which constitutes a better gage length. As observed in Fig. 15, the average plastic strain field inside the secondary grooves builds up at their propagating tip (about $270 \mu \mathrm{m}$ ahead of the crack tip) and keeps rising over a $50 \mu \mathrm{m}$ region before saturating to large values of about $450 \%\left(\varepsilon_{H}=1.7\right)$ with no further evolution during the approach of the propagating crack tip. The unstrained width $h_{S G}$ of the secondary groove

${ }^{6}$ Displacement $\Delta u_{x}$ between points A and B in Fig. 14 was measured to be $45 \pm 90 \mathrm{~nm}$ during the whole crack propagation and formation of the groove 

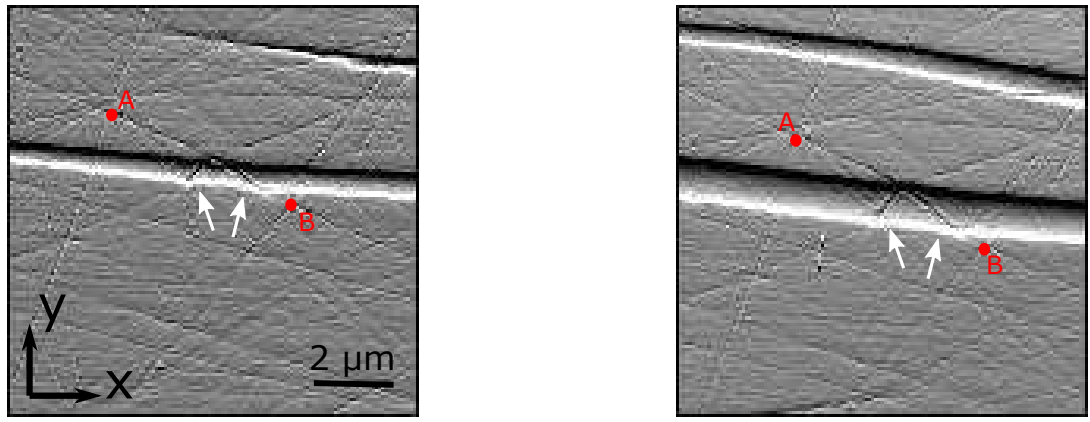

Figure 14: AFM observation in error signal mode of the abrupt tilt of initially straight polishing lines when they come across a secondary groove (as indicated by withe arrows). Left and right images present the same area at two different times (separated by 3 hours) of the formation of the secondary groove. Points A and B are visual indicators located at polishing lines intersections on opposite sides of the groove to help visualisation of the polishing lines tilt. It can be noticed that during crack propagation and development of the groove, $x_{B}-x_{A}$ remains nearly constant.

can be estimated by subtracting to the initial unstrained length $L_{0}$ of the vertical segment, the length $L_{\text {out }}$ of the unstrained portions of the segment that are out of the secondary grove. The unstrained width $h_{S G}$ progressively increases during the development of the secondary groove in its first $50 \mu \mathrm{m}$ portion. However, due to the limited resolution of the images, an accurate estimate of the length $L_{\text {out }}$ and thus of the unstrained width could only be performed for the final value corresponding to the fully developed secondary groove: $L_{\text {out }}=1.8 \mu \mathrm{m}, h_{S G}=0.3 \mu \mathrm{m}$. The stretched length of the groove is $1.35 \mu \mathrm{m}$. Since the values reported in Fig. 15 are obtained by simply dividing the measured displacement difference $\Delta u_{y}$ by this constant estimation $h_{S G}$, the transient increase in the first $50 \mu \mathrm{m}$ should only be interpreted in terms of displacement difference $\Delta u_{y}(X)$, while the average strain is expected to be spatially constant according to the previous observations on the tilt of the polishing lines. The displacement difference $\Delta u_{x}$ on the same vertical segment crossing the secondary groove was negligible within experimental incertitudes, which reveals the absence of shear on the secondary grooves. Once again the 2D projection of the average strain field is essentially a uniaxial extension perpendicular to the propagation axis.

We remark that the features of the strain field inside the secondary grooves are quite different from the main necking region. Both the spatial homogeneity and the large values of the plastic strain field in the secondary bands are consistent with what is expected for crazes.

However, these secondary crazes are not directly associated with the main 


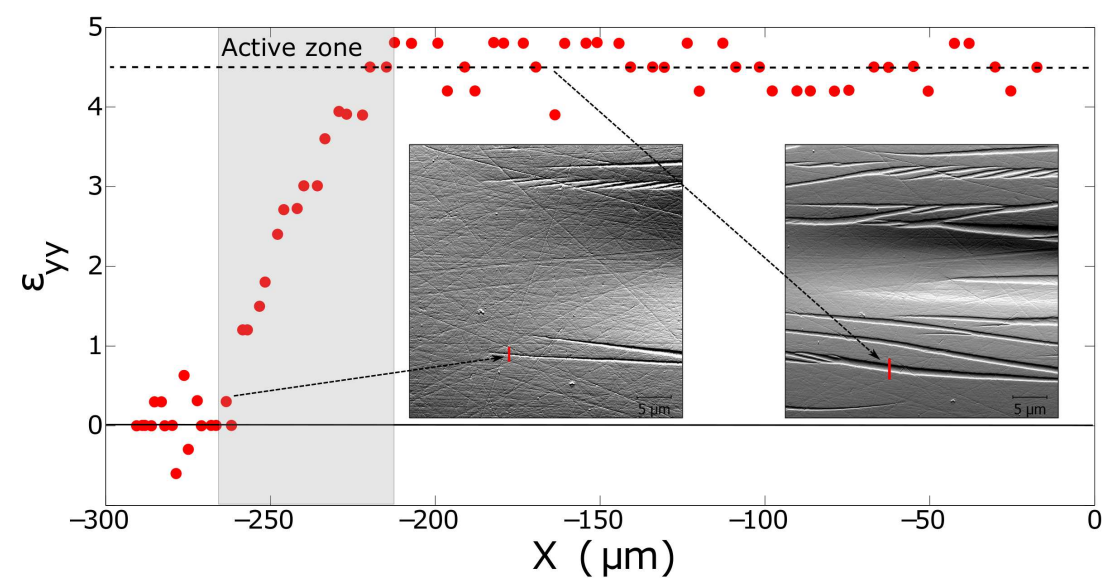

Figure 15: In plane nominal strain field in the direction normal to the crack as a function of the distance $X$ from the crack tip, calculated along a secondary groove as indicated in the inserts.

crack propagation through the whole thickness of the sample. They rather propagate from the free surface of the sample towards the interior, driven by the local stretching field originating from the main necking as argued above. Their lateral propagation along the crack direction $x$ is thus simply caused by the propagation of the necking field ahead of the main crack. The depth of penetration of these secondary crazes in the sample in direction $z$ can be inferred to amount to a few microns by considering both the average distance between them and their mutual interaction during propagation when they approach to smaller distances. This order of magnitude is also consistent with the $10 \mu \mathrm{m}$ depth of the surface layer affected by the main necking region, which is the cause for these secondary grooves.

The few micron propagation distance of these secondary surface crazes towards the interior of the sample (direction $z$ ) is less than the typical $30 \mu \mathrm{m}$ craze length observed by Doll (1983) during bulk crack propagation (direction $x$ ). This provides a sound explanation for the absence of fractures inside these secondary grooves. In other words, these secondary crazes propagate towards the inner part of the sample over too short a distance to nucleate a fracture. They thus remain arrested by the stress gradient within the depth as "unaccomplished" surface crazes that decorate the neighborhood of the main necking region at the free surface of the sample, without major consequences on the propagation of the main crack in the bulk.

In the main crack the craze propagation close to the free surface is hampered 


\section{Perspective for future work}

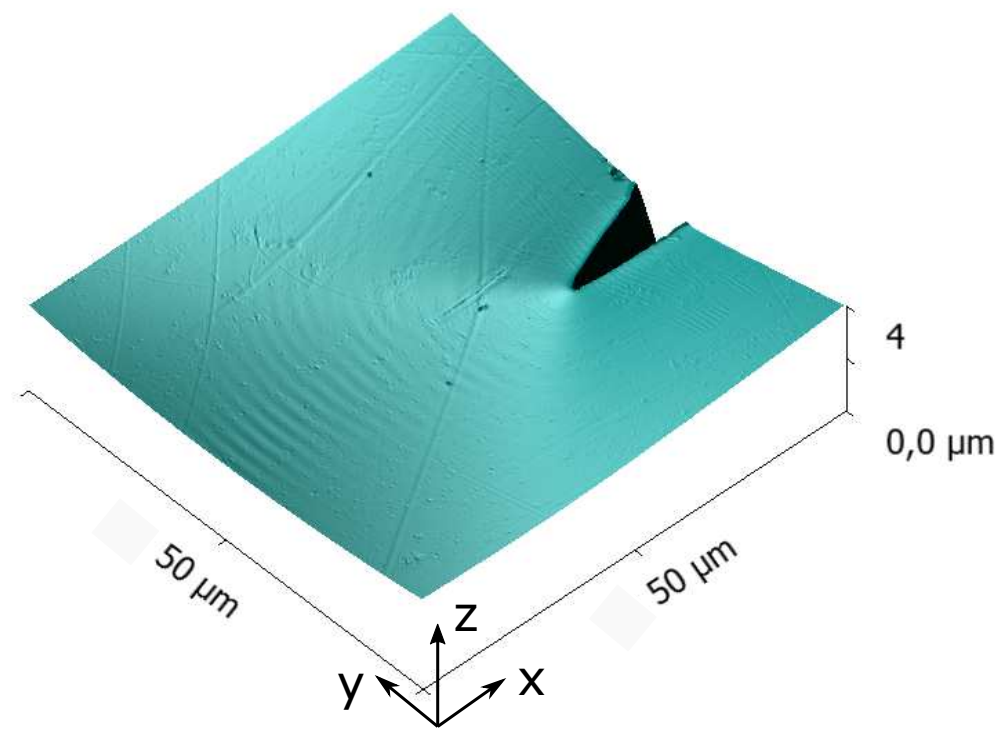

Figure 16: 3D representation of the topography of the crack tip region $\left(50 \times 50 \mu \mathrm{m}^{2}\right)$ measured by AFM at the free surface of an epoxy resin sample.

by the reduction of stress triaxiality, leading to the formation of the main surface necking as discussed in section 4.2. On the contrary, the presence of these secondary crazes at the free surface can be justified since their direction of propagation is different. On the one hand, the craze associated to the main crack propagates in the direction $x$ parallel to the free surface and the associated 2D crack tip field (in the $x-y$ plane) undergoes a change in triaxiality while approaching the free surface. On the other hand, the secondary surface crazes propagate in the direction $z$ that is perpendicular to the free surface and the 2D strain field associated to their propagating front (in the $y-z$ plane) is essentially in plane-strain since it does not approach to any free surface when moving along a direction orthogonal to its plane. Moreover, the stress triaxiality at their propagation region is locally enhanced by the stretch induced by the formation of the main valley.

In order to appreciate the high potential of this novel technique in discriminating the deformation mechanisms in the process zone for different glassy polymers, Fig. 16 shows a preliminary result on the in situ AFM imaging of fracture propagation in a model epoxy resin. When compared to Fig. 8, the surface displacement 
field in the process zone region of the epoxy resin appears to be very different. The absence of both the very extended surface necking region and of the secondary grooves are to be noticed. The surface displacement field rather appears as a very smooth shear yielding field with a clear strain concentration towards the crack tip. This process zone mechanism is essentially the same as the one expected for the crack propagation in the bulk, even if probably enhanced in size due to the surface loss of triaxiality (Williams, 1984). The absence of crazing in epoxies is generally attributed to the high crosslinking density (Halary et al., 2011). In the presence of such a smooth strain field, the present technique can be enriched by the possibility of computing the full strain field by digital image correlation.

\section{Conclusion}

A very promising novel technique is presented for investigating the mechanisms of crack propagation in glassy polymers and measuring the strain fields at the scale of their micrometric process zone during steady-state propagation by atomic force microscopy. The first important required achievement is the ability to properly initiate and propagate a very well conditioned mode I fracture in a glassy polymer (PMMA) and to ensure a stable steady-state condition over several days in order to allow for an extensive campaign of AFM observations. The second achieved step is to obtain a valuable estimation of the crack driving conditions (in terms of a stress intensity factor $K_{I}$ ) even when the DCDC sample undergoes some bulk plastic yielding in the central hole region due to is low strength/toughness ratio. This is assessed by the agreement of the measured $K_{I c}(v)$ crack propagation curves with the ones reported in the literature. These two achievements are a necessary condition to link the microscopic scale observations of the crack tip mechanisms with the macroscopic scale of the sample loading.

The acquisition of long AFM image series during several days of slow steadystate crack propagation allows for a rich and quantitative real-time evaluation of the damage mechanisms and the related strain fields at the free surface of the sample in a region that is progressively crossed by the crack tip process zone. While the out-of-plane displacement field is directly provided by the AFM topographic images, the in-plane displacement field can be obtained by tracking the relative motion of remarkable surface points.

The AFM observations presented for PMMA reveal a very rich and unexpected surface fracture pattern, which is quite different from the well known crazing behavior that occurs at the crack front in the bulk of the PMMA samples where plane-strain conditions are prevailing (Doll, 1983; Kramer, 1983). The analysis 
of the surface displacement field associated with crack propagation, together with post-mortem investigation by SEM, leads to the interpretation of these observations as a change of fracture mechanism from crazing in the bulk to shear yielding in a surface layer, where the prevailing plane-stress conditions involve a local reduction of stress triaxiality. The thickness of the layer affected by this change of mechanism is about $10 \mu \mathrm{m}$, which is the same order of magnitude as the Dugdale length in plane-strain as argued by Williams (1984). While changes in the process zone size are well known to occur in plane-stress surface layers (Williams, 1984), it is the first time to our knowledge that a clear observation of a global change of mechanisms is reported at the surface of a thick sample.

The surface shear necking region presents a ten fold increased length with respect to the craze in the bulk of the sample. The plastic strain field is shown to affect only half of this length and it presents a very smooth evolution up to $70 \%$ plastic extension close to the crack tip. This is comparable to the maximum plastic extension obtainable in uniaxial tensile tests on PMMA close to its glass transition temperature (G'Sell and Jonas, 1979). This observation is a nice proof that although PMMA is macroscopically brittle in tension at ambient temperature, it can be plastically cold drawn at scales smaller or comparable with the Dugdale length. The limiting extension is provided by the entanglement network, which still resists plastic flow before the material is broken.

The complex pattern of secondary grooves that develops along the main necking region can be attributed to secondary surface crazing by carefully analyzing the real-time development of the surface strain fields. These secondary surface crazes are shown to be formed in the first half of the main necking region, where only elastic tensile deformations are prevailing with a principal direction orthogonal to the fracture plane. The secondary crazes are shown to be nucleated on these polishing lines (cf. movie in the SI) that are more closely orthogonal to the surface tensile stress and which act as surface defects for nucleation. These secondary crazes are soundly propagating towards the bulk of the sample on a limited depth of about 10 microns, which corresponds to the region affected by the tensile stresses associated to the surface necking layer. This depth is consistent with the relative distance where these surface crazes are observed to mutually interact.

The preliminary images reported for an epoxy resin confirm the general applicability of the technique to different glassy polymers and allow to appreciate a very different organization of the plastic strain fields in the process zone. This novel technique is thus very promising to achieve important insights on the effect of the different macromolecular structures of glassy polymers on their fracture toughness. 


\section{Acknowledgements}

This work has been supported by the French ANR through grant PROMORPH ANR-2011-RMNP-006. We thank J.W. Hutchinson and C. Creton for fruitful discussions.

\section{References}

Argon, A.S., Hannoosh, J.G., 1977. Initiation of crazes in polystyrene. Phil. Mag. 36, 1195-1216.

Binnig, G., Quate, C.F., 1986. Atomic force microscopy. Phys. Rev. Lett. 56, 930933.

Brown, H.R., 1991. A molecular interpretation of the toughness of glassy polymers. Macromol. 24, 2752-2756.

Célarié F., Ciccotti M., Marlière, C., 2007. Stress-enhanced ion diffusion at the vicinity of a crack tip as evidenced by atomic force microscopy in silicate glasses. J. Non-Cryst. Solids. 353, 51-68.

Ciccotti, M., 2009. Stress-corrosion mechanisms in silicate glasses. J. Phys. D: Appl. Phys. 42, Art. N. 214006.

Doll, W., 1983. Optical interference measurements and fracture mechanics analysis of crack tip craze zones. Adv. Polym. Sci. 52/53, 105-168.

Donald, A.M., Kramer, E.J., 1982. The competition between shear deformation and crazing in glassy polymers. J. Mat. Sci. 17, 1871-1879.

Dugdale, D.S., 1960. Yielding of steel sheets containing slits. J. Mech. Phys. Solids 8, 100-104.

Fett, T., Rizzi, G., Munz, D., 2005. T-stress solution for DCDC specimens, Eng. Fract. Mech. 72, 145-149.

Griffith, A.A., 1920. The phenomena of rupture and flow in solids. Phil. Trans. R. Soc. Lond. A 221, 163-198.

Grimaldi, A., George, M., Pallares, G., Marlière, C., Ciccotti, M., 2008. The crack tip : a nanolab for studying confined liquids. Phys. Rev. Lett. 100, Art. N. 165505. 
G'Sell C., Jonas J.J., 1979. Determination of the plastic behaviour of solid polymers at constant true strain rate. J. Mat. Sci. 14, 583-591.

Han, K., Ciccotti, M., Roux, S., 2010. Measuring nanoscale stress intensity factors with an atomic force microscope. EPL. 89, Art. N. 66003.

Halary, J.L., Lauprètre, F., Monnerie, L., 2011. Polymer Materials, John Wiley \& Sons, Haboken, New Jersey.

He, M.Y., Turner, M.R., Evans A.G., 1995. Analysis of the double cleavage drilled compression specimen for interface fracture energy measurements over a wide range of mode mixities. Acta Metallur. Mater. 43, 3453-3458.

Hutchinson, J.W., Suo, Z., 1992. Mixed mode cracking in layered materials, Adv. Appl. Mech. 29, 63-191.

Idonije, K., Motuku, M., Shehata, I., Aglan, H., 1993. Evaluation of the stress intensity factor of brittle polymers based on the crack arrest concept, J. Reinf. Plast. 12, 778-786.

Janssen, C., 1974. Specimen for fracture mechanics studies on glass. Proc. 10th Int. Cong. on Glass (Kyoto, Japan) pp. 10.23-10.30.

Kramer, E.J., 1983. Microscopic and molecular fundamentals of crazing, Adv. Polym. Sci. 52/53, 1-56.

Kramer, E.J., Berger, 1990. Fundamental Processes of Craze Growth and Fracture. Adv. Polym. Sci. 91/92, 1-68.

Lawn, B.R., 1993. Fracture of Brittle Solids, second ed. Cambridge University Press, Cambridge.

Lemaitre, J., Chaboche, J.L., 1990. Mechanics of Solid Materials, first ed. Cambridge University Press, Cambridge.

Marshall, G.P., Coutts, L.H., Williams, J.G., 1974. Temperature effects in the fracture of PMMA, J. Mater. Sci. 9, 1409-1419.

Marshall, G.P., Williams, J.G., 1973. The correlation of fracture data for PMMA. J. Mater. Sci. 8, 138-140. 
McClintock, F.A., Irwin, G.R., 1964. Plasticity aspects of fracture mechanics. In: Fracture toughness testing and its applications, ASTM STP 381, Philadelphia, 84-113.

Michalske, T.A., Smith, W.L., Chen, E.P., 1993. Stree intensity calibration for the double cleavage drilled compression specimen, Eng. Fract. Mech. 45, 637-642.

Monnerie, L., Halary, J.L., Kausch, H.H., 2005. Deformation, Yield and Fracture of Amorphous Polymers: Relation to the Secondary Transitions, Adv. Polym. Sci. 187, 215-364.

Nziakou, Y., 2015. Analyse multi-échelle des mécanismes d'endommagement des matériaux composites à morphologie complexe destinés à l'aéronautique, $\mathrm{PhD}$ thesis, Université Pierre et Marie Curie, Paris, France.

Pallares, G., Ponson, L., Grimaldi, A., George, M., Prevot, G., Ciccotti, M., 2009. Crack opening profile in DCDC specimen. Int. J. Fract. 156, 11-20.

Pallares, G., Grimaldi, A., George, M., Ponson, L., Ciccotti, M., 2011. Quantitative analysis of crack closure driven by Laplace pressure in silica glass. J. Am. Ceram. Soc. 94, 2613-2618.

Pallares, G., George, M., Ponson, L., Chapuliot, S., Roux, S., Ciccotti, M., 2015. Multiscale investigation of stress-corrosion crack propagation mechanisms in oxide glasses. Corr. Rev. 33, 501-514.

Plaisted, T.A., Amirkhizi, A.V., Nemat-Nasser, S., 2006. Compression-induced axial crack propagation in DCDC polymer samples: experiments and modelling. Int. J. Fract. 141, 447-457.

Réthoré, J., Estevez, R., 2013. Identification of a cohesive zone model from digital images at the micron-scale. J. Mech. Phys. Solids, 61, 1407-1420.

Si, L., Massa, M.V., Dalnoki-Veress, K., Brown, H.R., Jones, R.A., 2005. Chain entanglement in thin freestanding polymer films. Phys. Rev. Lett. 94, Art. no. 127801 .

Williams, J.G., 1984. Fracture Mechanics of Polymers, Ellis Horwood, Chichester. 